\title{
Expression of interferon regulatory factor 5 is regulated by the $\mathrm{Sp1}$ transcription factor
}

\author{
JIN SHU $^{1}$, XIAO-HUA WANG ${ }^{1}$, LAN-BO ZHOU $^{2}$, CHUN-MING JIANG $^{1}$, \\ WEI-XIA YANG ${ }^{1}$, RUI JIN ${ }^{1}$, LU-LU WANG ${ }^{1}$ and GUO-PING ZHOU ${ }^{1}$ \\ ${ }^{1}$ Department of Pediatrics, The First Affiliated Hospital; ${ }^{2} 2013$ Clinical Class 7, School of Basic Medical Sciences, \\ Nanjing Medical University, Nanjing, Jiangsu 210029, P.R. China
}

Received August 3, 2015; Accepted July 20, 2016

DOI: $10.3892 / \mathrm{mmr} .2016 .5565$

\begin{abstract}
The transcription factor, interferon regulatory factor 5 (IRF5), is important in the induction of type I interferon, proinflammatory cytokines and chemokines, and is involved in autoimmune diseases and tumourigenesis. However, the mechanisms underlying the transcriptional regulation of wild-type IRF5 remain to be fully elucidated. The present study was primarily designed to clarify whether specificity protein 1 (Sp1) was involved in the regulation of IRF5. Initially, the IRF5 promoter region was cloned and its promoter activity was examined using Hela and HEK 293 cells. Deletion analyses revealed that the region spanning -179 to +62 was the minimal promoter of IRF5. Bioinformatics analyses showed that this region contained three putative Sp1 binding sites, and mutational analyses revealed that all the $\mathrm{Sp} 1$ sites contributed to transcriptional activity. Secondly, the overexpression of Sp1 was found to increase the activity of the IRF5 promoter and the mRNA level of IRF5, determined using reporter gene assays and polymerase chain reaction analysis, respectively. By contrast, treatment with mithramycin and Sp1 small interfering RNA significantly reduced the activity of the IRF5 promoter and the mRNA level of IRF5. Finally, the results of an electrophoretic mobility shift assay and a chromatin immunoprecipitation assay demonstrated that Sp1 bound to the promoter region of IRF5 in vitro and in vivo. These results
\end{abstract}

Correspondence to: Professor Guo-Ping Zhou, Department of Pediatrics, The First Affiliated Hospital, Nanjing Medical University, 300 Guangzhou Road, Nanjing, Jiangsu 210029, P.R. China E-mail: gpzhou2013@126.com

Abbreviations: BSA, bovine serum albumin; ChIP, chromatin immunoprecipitation; DMEM, Dulbecco's modified Eagle's medium; EMSA, electrophoretic mobility shift assay; FBS, fetal bovine serum; IRF5, interferon regulatory factor 5; siRNA, small interfering RNA; SLE, systemic lupus erythematosus; SNP, single-nucleotide polymorphism; Sp1, specificity protein 1; TSS, transcription start site

Key words: interferon regulatory factor 5, transcriptional activity, promoter, specificity protein 1 , mithramycin suggested that the Sp1 transcription factor is the primary determinant for activating the basal transcription of the IRF5.

\section{Introduction}

The human interferon regulatory factor (IRF) family comprises nine cellular IRFs, each of which has pleiotropic biological functions (1). IRF5 is important in the induction of type I interferons (IFNs), proinflammatory cytokines and chemokines. Therefore, it is involved in innate and adaptive immunity (2-5). There is increasing evidence showing that IRF5 occupies a prominent place among the genetic factors involved in the susceptibility to human systemic lupus erythematosus (SLE), Sjögren's syndrome and rheumatoid arthritis (6-8). Previous studies have demonstrated that the expression, alternative splicing and protein levels of IRF5 were significantly elevated in primary purified peripheral blood mononuclear cells from patients with SLE $(6,9)$.

As a significant factor mediating autoimmune diseases, IRF5 exhibits certain tumour-suppressor properties, as it can induce p21, B cell lymphoma 2-antagonist/killer 1, B cell lymphoma 2-associated X protein and Caspase 8 (10-12). The ectopic expression of IRF5 reduces the proliferation of chronic myeloid leukaemia cells in vitro as a target of BCR-ABL kinase (13). The expression of IRF5 is reduced in gastric cancer, and is associated with progression and metastasis of breast cancer $(14,15)$. By contrast, the tumour-promoting effect of IRF5 has also been reported. IRF5 is expressed at high levels in primary and immortalised thyroid carcinoma, but not in normal thyrocytes, whereas ectopic expression of IRF5 increases the proliferation of malignant thyroid cells (16). A high expression level of IRF5 is specific to Hodgkin's lymphoma cells and is crucial for their survival (17). Different functions of IRF5 have been reported functions, as it exists as multiple alternatively spliced variants, each with distinct cell type-specific expression and function (18).

In addition, genetic polymorphisms in IRF5, which lead to an increase in its expression, are associated with inflammatory and autoimmune diseases. Rs2004640, the first single-nucleotide polymorphism (SNP) to be identified, is associated with the elevated expression of multiple isoforms of IRF5, and is an important genetic risk factor for SLE (19). Another SNP 
(rs77571059, CGGGG indel) is found 64 bp upstream of the transcription start site (TSS) for exon 1A, which has four copies (4x) of the CGGGG repeat sequence. The CGGGG 4x variant allows additional binding of the Sp1 transcription factor and is associated with the increased expression of IRF5 (20). Sp1 was the first among all the transcription factors to be identified and cloned, and is responsible for the transcription of several mammalian and viral genes with abundant GC boxes in their promoter region through three $\mathrm{C} 2 \mathrm{H} 2$-type zinc fingers in the C-terminal domain (21). However, the transcriptional regulation of IRF5, and whether Sp1 can regulate the expression of IRF5 remains to be fully elucidated.

In the present study, it was determined that the exogenous expression of $\mathrm{Sp} 1$ led to a significant increase in promoter activity and the mRNA expression of IRF5. These findings suggested that $\mathrm{Sp} 1$ positively regulated the transcription of IRF5 through binding to the minimal promoter region of IRF5 and indicated a potential application of $\mathrm{Sp} 1$ as a target for treatment of IRF5 related diseases.

\section{Materials and methods}

Cell culture and reagents. Human embryonic kidney (HEK) 293 and Hela cells were obtained from American Type Culture Collection (Manassas, VA, USA). The cells were maintained in Dulbecco's high glucose modified Eagle's medium (DMEM) with $10 \%$ heat-inactivated fetal bovine serum (FBS), supplemented with penicillin $(100 \mathrm{U} / \mathrm{ml})$ and streptomycin $(100 \mu \mathrm{g} / \mathrm{ml})$. Mithramycin was purchased from Sangon Biotech (Shanghai, China). Bovine serum albumin (BSA) was purchased from Santa Cruz Biotechnology, Inc. (Dallas, TX, USA).

Plasmids and small interfering RNA (siRNA). The DNA sequence $(-1,760$ to +62$)$ of the IRF5 promoter region was amplified by polymerase chain reaction (PCR) and digested with KpnI and BglII (Thermo Fisher Scientific, Inc., Waltham, MA, USA), followed by being subcloned into the promoter-less luciferase expression plasmid, pGL3-Basic (Promega Corp., Madison, WI, USA). The resulting plasmid was termed pGL-1760/+62. Truncated plasmids of the IRF5 promoter were constructed using pGL-1760/+62 as a template. The potential transcription binding sites for Sp1 were identified using online software TFSEARCH version 1.3 (www.cbrc.jp/research/db/TFSEARCH.html) and JASPAR database version 5.0 (jaspar.genereg.net). A series of mutant plasmids of pGL-179/+62 carrying a number of nucleotide substitutions (Mut-Sp1-A, Mut-Sp1-B Mut-Sp1-C, Mut-Sp1-A+B, Mut-Sp1-A+C, Mut-Sp1-B+C and Mut-Sp1-A+B+C) were constructed using a site-directed mutagenesis kit (Takara Bio, Inc., Otsu, Japan). The names of the plasmids and corresponding olignucleotides are shown in Table I. The pN3-empty and pN3-Sp1 expression plasmids were donated by Dr Guntram Suske (University of Marburg, Marburg, Germany). The siRNAs were synthesised and high performance purified (GenePharma, Shanghai, China). The targeted sequence used to silence specific gene transcription was sense 5'-AUCACUCCAUGGAUGAAAUGATT-3' for Sp1. The sequence of the control siRNA was 5'-UUCUCC GAACGUGUCACGUTT-3'.
Transient transfection and luciferase assays. Transient transfection of the HEK 293 and Hela cells was performed using Lipofectamine $^{\mathrm{TM}} 3000$ (Invitrogen; Thermo Fisher Scientific, Inc.), according to the manufacturer's protocol. The cells were seeded into 96 -well plates $\left(1.5 \times 10^{4} /\right.$ well $) 24 \mathrm{~h}$ prior to transfection. For the Sp1 siRNA or Sp1 overexpression experiments, reporter plasmids containing siRNA for Sp1 or the Sp1 expression plasmid were cotransfected into the cells and harvested after $24 \mathrm{~h}$. For the mithramycin experiment, $24 \mathrm{~h}$ following transfection with the reporter plasmids, the cells were treated with mithramycin $(100 \mathrm{nM})$ or distilled water for $24 \mathrm{~h}$ to measure luciferase activity. Luciferase activity was detected using the Dual Reporter assay system (Promega Corp.). All experiments were performed independently in triplicate.

Electrophoretic mobility shift analysis (EMSA). Nuclear extracts were obtained using nuclear and cytoplasmic extraction reagents (Thermo Fisher Scientific, Inc.), according to the manufacturer's protocol. As the Sp1-A and Sp1-B binding sites overlapped, probe A comprised these two binding sites (-85 to -48: 5'-AGTGGATTCGCGGGGCGGGGCGGG GCACTGCCCGCGC-3') labeled with biotin, and probe B contained the Sp1-C binding site (-36 to -6: 5'-AGCAGCTGC CCAGGGGCGGGGGCGGCAAGA-3'). The nuclear protein extract $(10 \mu \mathrm{g})$ was incubated in binding buffer for $10 \mathrm{~min}$ at room temperature, following which the biotinylated probes were added, and the reaction mixture was incubated at room temperature for another $20 \mathrm{~min}$. For the competition experiments, unlabeled probes were added to the reaction mixture at 100 -fold excess molar concentration. In the supershift assays, $1.5 \mu \mathrm{g}$ of Sp1 antibody (cat. no. sc-59; 1:200; Santa Cruz Biotechnology, Inc., Santa Cruz, CA, USA) was preincubated for $20 \mathrm{~min}$ at room temperature. The binding reactions were assessed by electrophoresis through a $6 \%$ polyacrylamide gel, run for $1 \mathrm{~h}$ at $100 \mathrm{~V}$, followed by transfer onto a nylon membrane at $300 \mathrm{~mA}$ for $30 \mathrm{~min}$, cross-linking for 5 min under UV light, and detection with biotin-labeled DNA, according to the protocol of the Light Shift Chemiluminescent EMSA kit (Pierce Biotechnology, Inc., Rockford, IL, USA).

Chromatin immunoprecipitation (ChIP) assay. A ChIP assay was performed using the EZ-Magna Chip ${ }^{\mathrm{TM}}$ A kit (cat. no. 17-408; EMD Millipore, Billerica, MA, USA) according to the manufacturer's protocol. A total of $1 \times 10^{7}$ Hela cells were fixed in $1 \%$ formaldehyde at room temperature for $10 \mathrm{~min}$. The cell lysates were sonicated to generate 200-1,000 bp DNA fragments. The chromatin was immunoprecipitated using anti-acetyl histone $\mathrm{H} 3$ antibody (from kit; 1:100; EMD Millipore), anti-rabbit IgG antibody (from kit; 1:100; EMD Millipore) and anti-Sp1 antibody (1:50; Santa Cruz Biotechnology, Inc.). Following reverse cross-linking and DNA purification, DNA from the input and immunoprecipitated samples was assayed using semi-quantitative PCR with the following primers: Forward 5'-TGGCCCGAGGCT CAGCCCGGATCT-3' and reverse 5'-TCCGCCAACCTG CCGGGCACTTCC-3'. The PCR products were analysed using $2 \%$ agarose gel electrophoresis.

$q P C R$. Total RNA of the HEK 293 cells was extracted using TRIzol reagent (Invitrogen; Thermo Fisher Scientific, Inc.) 
Table I. Oligonucleotide sequences used for the generation of reporter constructs.

\begin{tabular}{|c|c|}
\hline Plasmid & Primer sequence $\left(5^{\prime}-3^{\prime}\right)$ \\
\hline PGL-1760 & $\begin{array}{l}\text { Sense: 5'-CGGGGTACCCTACCCATTCACATTTTCCCCATCC-3' } \\
\text { Antisense: 5'-GGAAGATCTGGGACCAAGCTGAGCTCTGC-3' }\end{array}$ \\
\hline PGL-1080 & $\begin{array}{l}\text { Sense: 5'-CGGGGTACCTACACCTGCTGCCTGTTGACCAAT-3' } \\
\text { Antisense: 5'-GGAAGATCTGGGACCAAGCTGAGCTCTGC-3' }\end{array}$ \\
\hline PGL-503 & $\begin{array}{l}\text { Sense:5'-CGGGGTACCCAGGGTTTGAGGATGAGAAAGGCAC-3' } \\
\text { Antisense: 5'-GGAAGATCTGGGACCAAGCTGAGCTCTGC-3' }\end{array}$ \\
\hline PGL-179 & $\begin{array}{l}\text { Sense: 5'-CGGGGTACCAGGGCACCGCGCCGTCTGGCATCTC-3' } \\
\text { Antisense: 5'-GGAAGATCTGGGACCAAGCTGAGCTCTGC-3' }\end{array}$ \\
\hline PGL-40 & $\begin{array}{l}\text { Sense: 5'-CGGGGTACCAGCAGCAGCTGCCCAGGGGCGG-3' } \\
\text { Antisense: 5'-GGAAGATCTGGGACCAAGCTGAGCTCTGC-3' }\end{array}$ \\
\hline PGL-9 & $\begin{array}{l}\text { Sense: 5'-CGGGGTACCAGACGCGGAAGTGCCCGGCAG-3' } \\
\text { Antisense: 5'-GGAAGATCTGGGACCAAGCTGAGCTCTGC-3' }\end{array}$ \\
\hline Mut-Sp1-A & $\begin{array}{l}\text { Sense: 5'-ATTCGCGGTTTTGGGCGGGGCACTGCC-3' } \\
\text { Antisense: 5'-CCACTCCGGGCCCCGCACTGACCTG-3' }\end{array}$ \\
\hline Mut-Sp1-B & $\begin{array}{l}\text { Sense: 5'-GGGCGGGGTTTGGCACTGCCCGCGCCCGGAG-3' } \\
\text { Antisense: 5'-CGCGAATCCACTCCGGGCCCCGCACTG-3' }\end{array}$ \\
\hline Mut-Sp1-C & $\begin{array}{l}\text { Sense: 5'-TGCCCAGGTTTTGGGGCGGCAAGACGCGGAAG-3' } \\
\text { Antisense: 5'-GCTGCTGCTGAGCTCCGGGCGCGGGCAG-3' }\end{array}$ \\
\hline Mut-Sp1-A+B & $\begin{array}{l}\text { Sense: 5' TTGGGTTTGGCACTGCCCGCGCCCGGAG-3' } \\
\text { Antisense: 5'AACCGCGAATCCACTCCGGGCCCCGCAC-3' }\end{array}$ \\
\hline Mut-Sp1-A+C & $\begin{array}{l}\text { Sense: 5' ATTCGCGGTTTTGGGCGGGGCACTGCC-3' } \\
\text { Antisense: 5'CCACTCCGGGCCCCGCACTGACCTG-3' }\end{array}$ \\
\hline Mut-Sp1-B+C & $\begin{array}{l}\text { Sense: 5'GGTTTGGCACTGCCCGCGCCCGGAGCTCAG-3' } \\
\text { Antisense: 5'CCGCCCCGCGAATCCACTCCGGGCCCCG-3' }\end{array}$ \\
\hline Mut-SP1-A+B+C & $\begin{array}{l}\text { Sense: 5' TTGGGTTTGGCACTGCCCGCGCCCGGAG-3' } \\
\text { Antisense: 5'AACCGCGAATCCACTCCGGGCCCCGCAC-3' }\end{array}$ \\
\hline
\end{tabular}

Restriction endonuclease sequences are shown in bold. Sp1, specificity protein 1 .

and then reverse transcribed use the PrimeScript RT Master Mix Perfect Real Time kit (Takara Bio, Inc.). qPCR analysis was performed with the Applied Biosystems Step One Plus Real-Time PCR system using SYBR Premix Ex Taq (Takara Bio, Inc.), the following thermocycling conditions were used: $95^{\circ} \mathrm{C}$ for $5 \mathrm{~min} ; 40$ cycles at $95^{\circ} \mathrm{C}$ for $15 \mathrm{sec}, 60^{\circ} \mathrm{C}$ for $1 \mathrm{~min}$. The specificity of amplification was assessed for each sample by melting curve analysis. The expression of IRF5 was normalised to GAPDH and the relative expression was calculated using the comparative $\mathrm{Cq}$ method (22). The primers used were as follows: IRF5, forward 5'-GGGCTT CAATGGGTCAACG-3' and reverse 5'-GCCTTCGGTGTA TTTCCCTG-3', and GAPDH, forward 5'-TGGTATCGT GGAAGGACTCATGAC-3' and reverse 5'-TGCCAGTGA GCTTCCCGTTCAGC-3'.

Statistical analysis. The results are expressed as the mean \pm standard error of the mean. Statistical analysis was performed using Student $t$-test with SPSS software (version 20.0; IBM SPSS, Armonk, NY, USA). P<0.05 was considered to indicate a statistically significant difference.

\section{Results}

IRF5 minimal promoter is located in the region between -179 and +62 . To identify the IRF5 minimal promoter (Fig. 1A), a series of luciferase reporter plasmids containing 5 ' deletions of the putative $(1,760 /+62)$ promoter region were cloned and transfected into Hela and HEK 293 cells (Fig. 1B and C). The luciferase assays revealed a 20-22-fold increase in the promoter activity of the pGL-1,760/+62, compared with the empty vector, indicating a functional promoter in the $-1,760 /+62$ region of IRF5. Deletion of the sequence between $-1,760$ and -179 increased the transcriptional activity by 1.6-2.0-fold, compared with pGL-1,760/+62 (Fig. 1B), suggesting negative regulatory elements were located in the $-1,760 /-179$ region. When the promoter was deleted to position -40 , transcriptional activity decreased significantly, by $69 \%$ in the Hela cells and $73 \%$ in the HEK 293 cells, compared with pGL-179/+62, which demonstrated that positive regulatory elements were located in the $-179 /-40$ region. Further 5' deletion in the -9 to +62-generated plasmid (pGL-9/+62) had a low level of promoter activity, indicating that the minimal promoter of IRF5 was located within the $-179 /+62$ region relative to the TSS. 
A

AGGGCACCGCGCCGTCTGGCATCTCCCTGGAGGCCCTGGGCCTG GCCCGAGGCTCAGCCCGGATCTGCAGTTGCCAGGTCAGTGCGGG
GCCCGGAGTGGATTCGCGGGGCGGGGCGGGGCACTGCCCGCGC
Sp1-A
CCGGAGCTCAGCAGCAGCTGCCCAGGGGCGGGGGCGGCAAGAC
$\underset{\text { Sp1-C }}{\text { TSS }}$ GCGAGTGCCGGCAGGTTGGCGGACCGGCGGAGGCGCAGC

CTGGGCAGAGCTCAGCTTGGTCCC

\section{B}
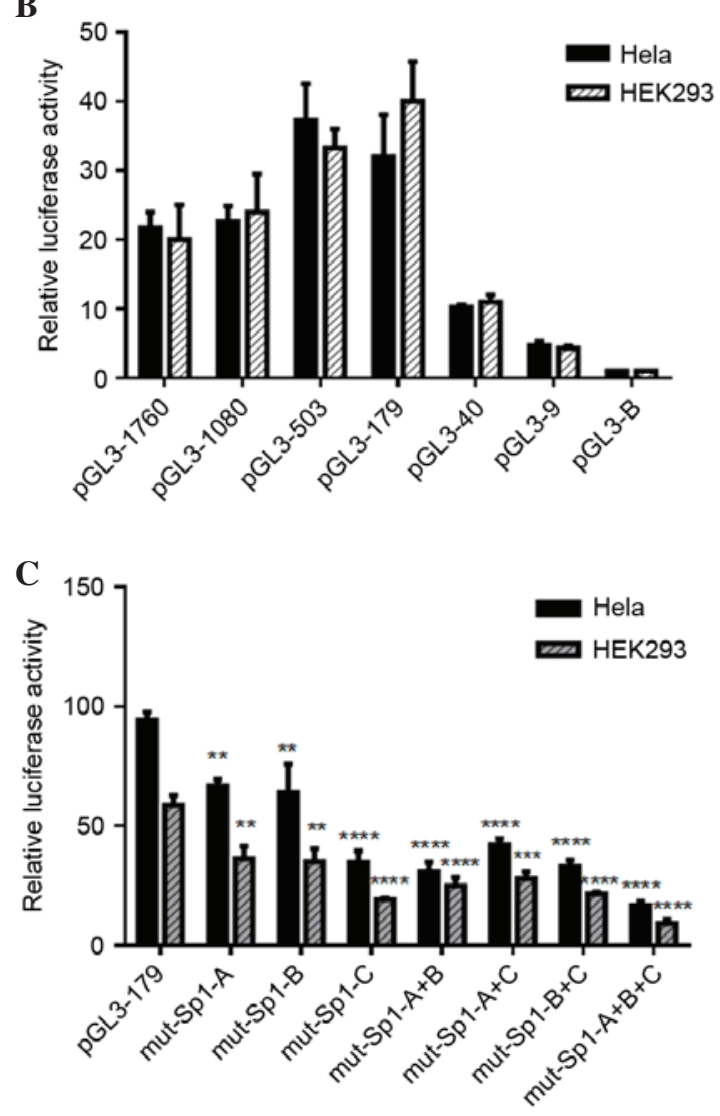

Figure 1. Characterization of the Sp1 binding sites in the IRF5 promoter. (A) Sequence and putative Sp1 binding sites of the minimal promoter of IRF5. The putative Sp1 binding sites (Sp1-A, Sp1-B and Sp1-C) are indicated at the regions highlighted. (B) Deletion analysis of the IRF5 promoter. Various IRF5 promoter plasmids were transfected into Hela and HEK 293 cell lines. After $24 \mathrm{~h}$, the cells were harvested for a luciferase assay. (C) Site-directed mutagenesis was performed using pGL-179/+62 as the template. Various constructs were transfected into HeLa and HEK 293 cells, and the luciferase activity was determined $\left({ }^{* *} \mathrm{P}<0.01,{ }^{* * *} \mathrm{P}<0.001\right.$ and $\left.{ }^{* * * *} \mathrm{P}<0.0001\right)$. IRF5, interferon regulatory factor 5 ; Sp1, specificity protein 1 ; TSS, transcription start site.

Sp1 is a transcriptional activator in the minimal promoter region of IRF5. The sequence between -179 and -40 upstream of the TSS had putative binding sites for Sp1, determined using online software TFSEARCH version 1.3 and JASPAR database version 5.0 (Fig. 1A). To investigate the role of these sites in the regulation of IRF5, a series of plasmids with 3-4 bp point mutations of Spl binding sites were constructed and transiently transfected into Hela and HEK 293 cells. As shown in Fig. 1C, mutations of Sp1-A, Sp1-B and Sp1-C in the Hela cells reduced the promoter activity to 71,68 and $37 \%$ of the pGL3-179/+62 promoter activity, respectively. Similarly, mutation of Sp1-A, Sp1-B and Sp1-C in in HEK 293 cells decreased the promoter activity to 62,60 and $33 \%$ of the pGL3-179/+62 promoter, respectively. However, combinatorial mutations (double mutations), had minimal additional effect on basal promoter activity. The triple mutation of $\mathrm{Spl}-\mathrm{A}+\mathrm{B}+\mathrm{C}$ reduced the promoter activity more markedly. To determine whether $\mathrm{Sp} 1$ directly regulated the transcription of IRF5, an Sp1-overexpression plasmid was introduced into Hela and HEK 293 cells. As shown in Fig. 2A and $\mathrm{B}$, the overexpression of $\mathrm{Sp} 1$ enhanced the luciferase activity of pGL3-179/+62 by 1-fold in the Hela cells and 4-fold in the HEK293 cells, respectively.

Silencing of Sp1 reduces IRF5 promoter activity. To further confirm the roles of $\mathrm{Sp} 1$ in the regulation of IRF5 promoter activity, the pGL-179/+62 plasmid was cotransfected together with Sp1 siRNA or si-control. As shown in Fig. 2C and $\mathrm{D}$, repression of the endogenous expression of $\mathrm{Sp} 1$ induced a marked decrease in promoter activity, by $57 \%$ in the Hela cells and 46\% in the HEK 293 cells, respectively. Mithramycin is known to bind to the GC box and inhibit Sp1 binding (23). Treatment with mithramycin also led to a significant decrease in promoter activity, by $63 \%$ in the Hela cells and 61\% in the HEK 293 cells, respectively (Fig. 2E and F).

Spl binds to the minimal promoter of IRF5 in vitro and in vivo. To validate whether Sp1 bound to the IRF5 promoter in vitro, an EMSA was performed. As shown in Fig. 3A and B, the nuclear extracts from the Hela cells bound to the labeled wild-type probe A and probe B, and formed shifted bands (lane 2). No shifted bands were observed when bovine serum albumin was substituted for the nuclear extracts (lane 1). The shifted bands were eliminated when incubated with 100 -fold excess unlabeled probe (lane 3). As shown in the lane 4, super-shifted bands were produced when incubated with Sp1 antibody, and the density of the corresponding shifted bands were reduced, indicating that $\mathrm{Sp} 1$ bound to multiple motifs in the IRF5 promoter in vitro. In addition, a ChIP assay was performed to examine whether Sp1 interacted with the IRF5 promoter in vivo. The cross-linked chromatin prepared from the Hela cells was immunoprecipitated with anti-acetyl histone H3, anti-IgG and anti-Sp1 antibodies. The DNA precipitated in the complexes was then subjected to PCR with primers covering the regions of the IRF5 promoter sequence. As shown in Fig. 3C, the anti-acetyl histone H3 and anti-Sp1 antibodies immunoprecipitated the sequence of the IRF5 promoter, whereas the non-specific IgG antibody failed to immunoprecipitate this sequence, suggesting that Sp1 was able to bind to the minimal promoter of IRF5 in vivo.

Sp1 regulates the mRNA expression level of IRF5. To further examine the role of $\mathrm{Sp} 1$ in regulating the expression of IRF5, siRNA, mithramycin or the Sp1-overexpression vector were transfected into HEK293 cells. The mRNA levels of IRF5 were detected using qPCR analysis. As shown in Fig. 4A and B, siRNA and mithramycin reduced the mRNA levels of IRF5 by 16 and $44 \%$, respectively. By contrast, the overexpression of Sp1 resulted in a 1.45-fold increase in the mRNA expression of IRF5, compared with the pN3-empty negative control (Fig. 4C), indicating that $\mathrm{Sp1}$ upregulated the mRNA expression level of IRF5. 
A

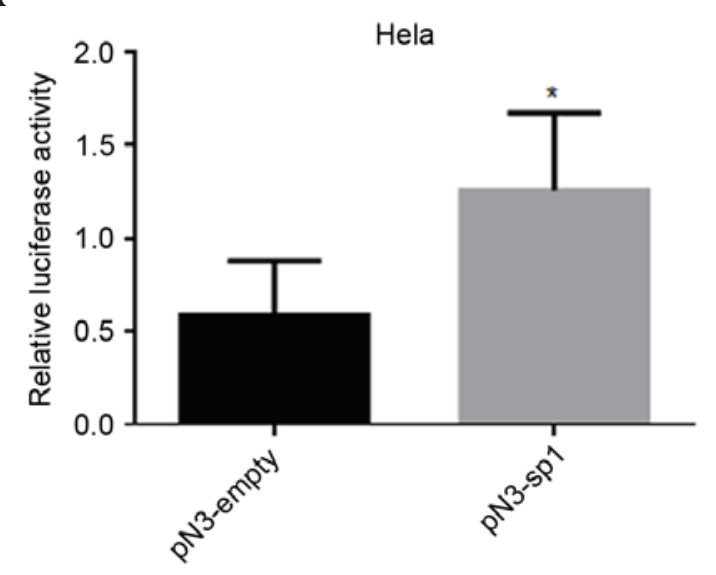

C

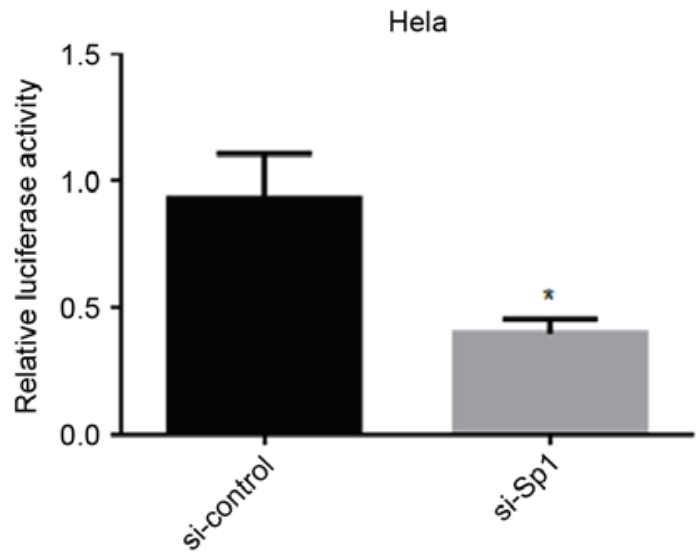

E

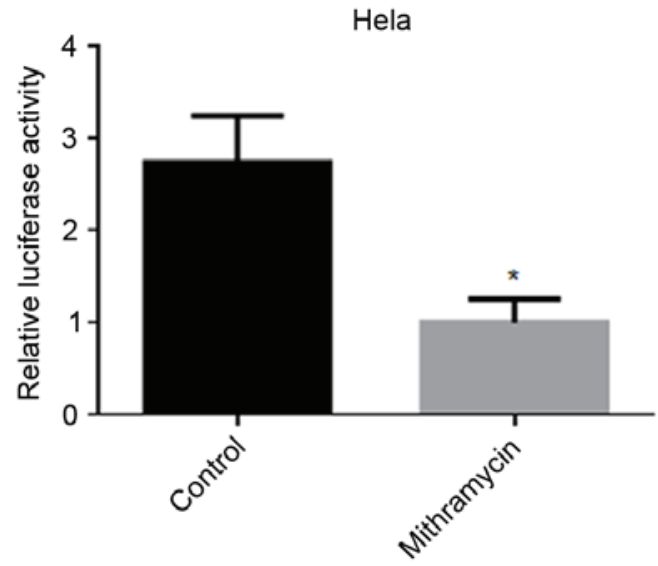

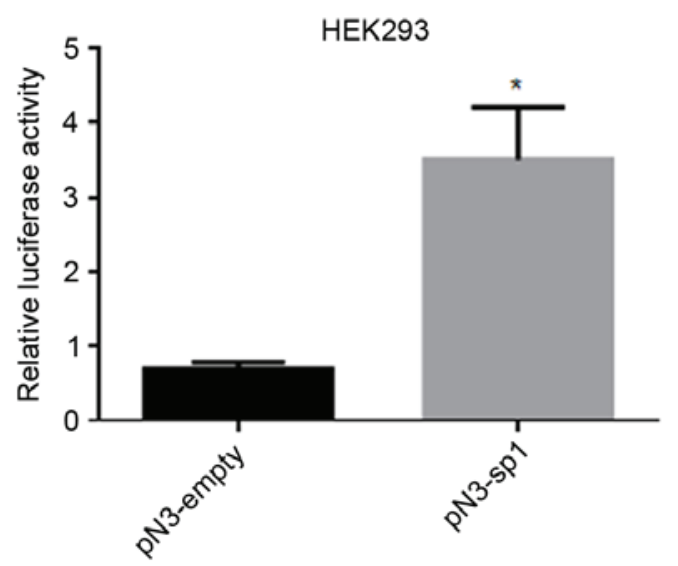

D

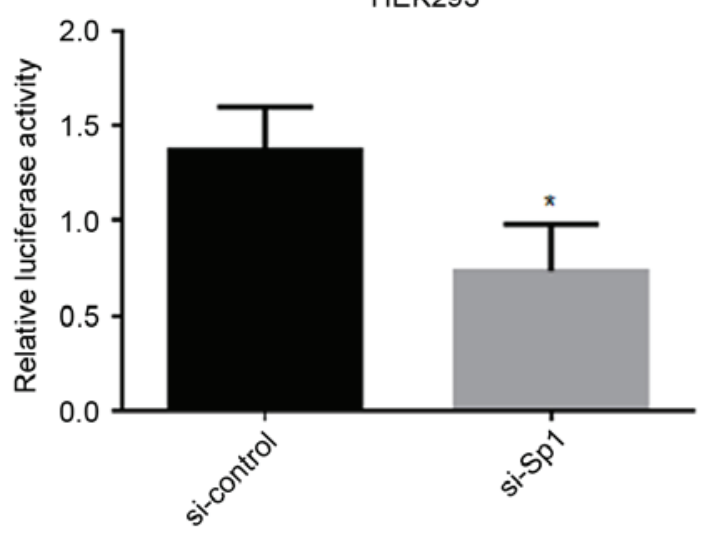

F

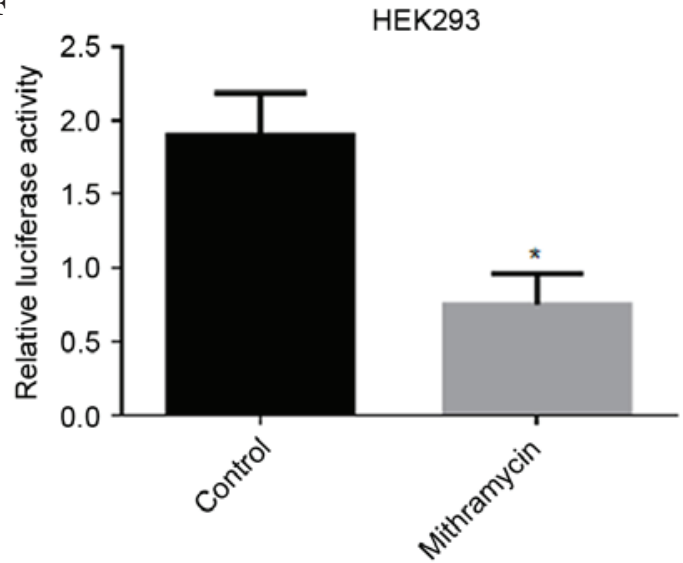

Figure 2. Regulation of IRF5 minimal promoter activity by Sp1 in Hela and HEK293 cells. Overexpression of Sp1 transactivated the human IRF5 promoter. (A) Hela and (B) HEK293 cells were cotransfected with pGL-179/+62 and pN3-empty or pN3-Sp1. Luciferase activity was determined 24 h following transfection ( $\mathrm{P}<0.05)$. Downregulation of Sp1 by siRNA reduced IRF5 promoter activity. (C) Hela and (D) HEK293 cells were cotransfected with pGL-179/+62 and Sp1-siRNA $(30 \mathrm{nM})$ or si-control $(30 \mathrm{nM})$. Luciferase activity was detected $24 \mathrm{~h}$ following transfection $\left({ }^{*} \mathrm{P}<0.05\right)$. Mithramycin treatment downregulates IRF5 promoter activity. (E) Hela and (F) HEK293 cells transfected with pGL-179/+62 were grown for $24 \mathrm{~h}$ and treated with mithramycin (100 nM) for another $24 \mathrm{~h}$, followed by analysis of luciferase activity $\left({ }^{*} \mathrm{P}<0.05\right)$. IRF5, interferon regulatory factor 5 ; Sp1, specificity protein 1 ; siRNA, small interfering RNA.

\section{Discussion}

In our previous studies, cloning of IRF3 and its spliced variant promoters was performed, and it was identified that $\mathrm{Sp} 1 / \mathrm{Sp} 3$ positively regulates the transcription of IRF3 through binding to the Sp1/NRF-1 binding site in the core promoter region of IRF3 $(24,25)$. The nine distinct alternatively spliced human IRF5 mRNAs (V1-V9) were previously identified by Mancl et al (18), who showed that V1 and V3 had distinct TSSs, and these two discrete promoters were cloned. V5 and V6 were 
A

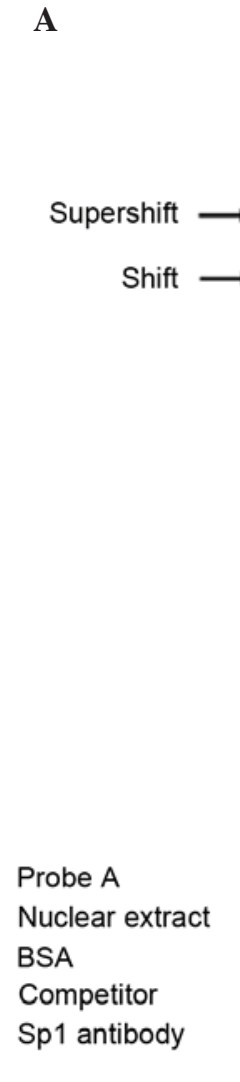

$\begin{array}{llll}1 & 2 & 3 & 4\end{array}$

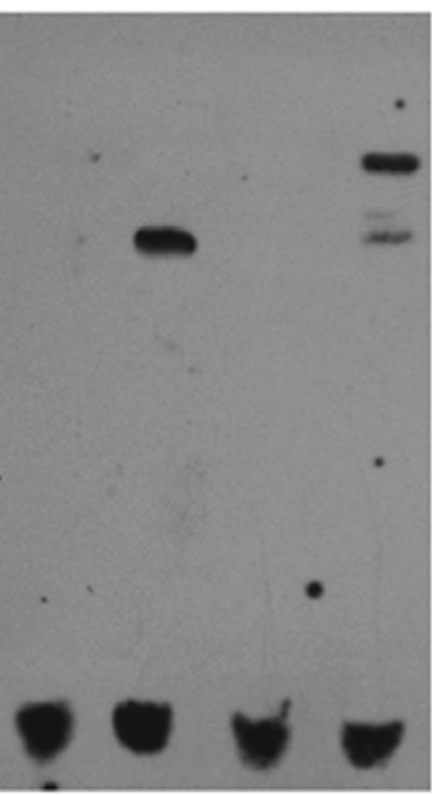

$+\quad+\quad+$

$+\quad+\quad+$

$+\quad-\quad-\quad+2 \quad+$

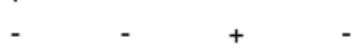

B

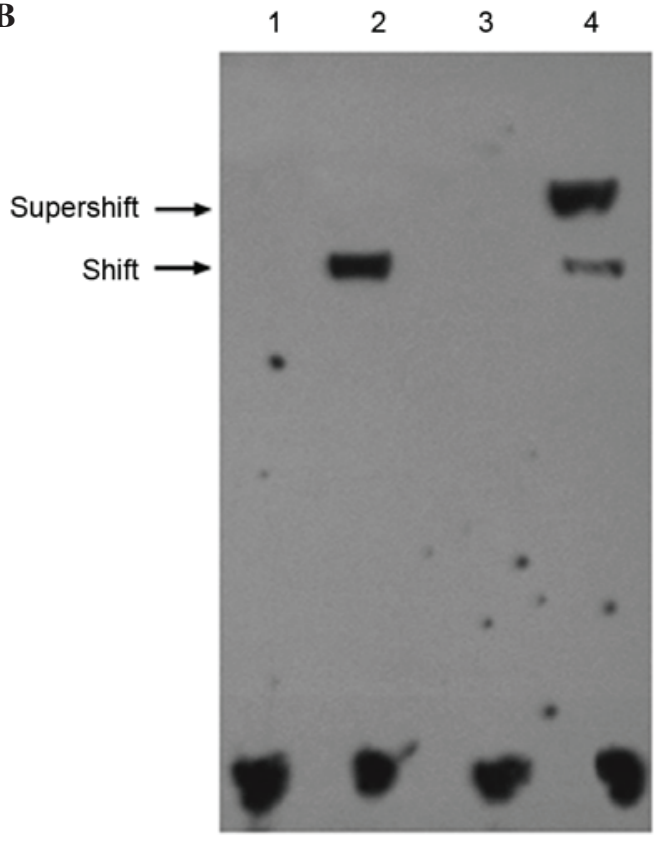

Probe B

Nuclear extract

BSA

Competitor

Sp1 antibody

C

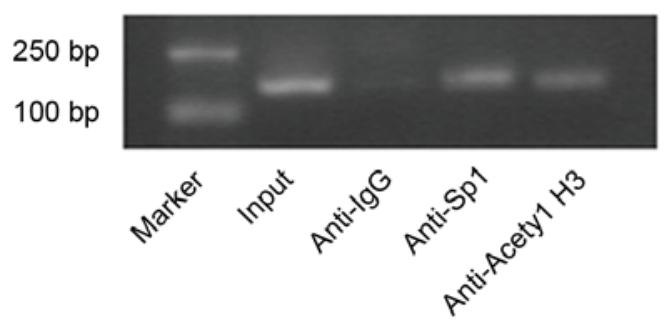

Figure 3. Sp1 specifically binds to the minimal promoter of IRF5. Electrophoretic mobility shift analysis showed Sp1 directly binding to multiple sites in the IRF5 promoter region in vitro. Nuclear protein extracts from the Hela cells were incubated with labeled (A) wild-type probe A or (B) probe B, containing Sp1 binding sites, in the presence of 100x competitor probe (lane 3) or in the absence of any competitor (lane 2). As a control, the nuclear extracts were substituted with BSA for the incubation with labeled wild-type probe A or probe B (lane 1). The super-shift assay was performed using anti-Sp1 antibody (lane 4). The arrows show shifted and super-shifted bands in lane 2 and lane 4, respectively. (C) A chromatin immunoprecipitation assay was used to examine the binding of Sp1 to the promoter of IRF5 in vivo. The anti-histone $\mathrm{H} 3$ antibody and anti-Sp1 antibody precipitated proteins bound in vivo to the amplified sequence of the IRF5 promoter, whereas anti-IgG antibody failed to precipitate proteins bound in vivo in this sequence. Polymerase chain reaction products (158 bp) were visualised using agarose gel electrophoresis and ethidium bromide staining. IRF5, interferon regulatory factor 5 ; Sp1, specificity protein 1; BSA, bovine serum albumin.

expressed in peripheral blood mononuclear cells, whereas $\mathrm{V7}$, V8 and V9 were expressed only in human cancer cells (18). In addition, Clark et al (26) revealed that IRF5 has four promoters for its four first exons: $1 \mathrm{~A}, 1 \mathrm{~B}, 1 \mathrm{C}$ and $1 \mathrm{D}$. These promoters were all found to contain Sp1 putative binding sites. When treated with imiquimod, a Toll-like receptor 7 ligand, the mRNA levels of IRF5 were doubled, and the 1A and 1D promoter activities were increased.

In the present study, the 5'-flanking region of the wild-type, IRF5, which had the most integrated reference sequence (V5), was cloned, which was located between the V1 and V3 promoter. The pGL-1760/+62 construct contained the 1A and $1 \mathrm{D}$ promoter without the $1 \mathrm{~B}$ or $1 \mathrm{C}$ promoter, however, the pGL-179/+62 construct contained only the 1A promoter, which possibly included the $4 \mathrm{x}$ variant of the CGGGG indel, but no Rs2004640. When subcloned into a luciferase reporter vector and transfected into Hela and HEK 293 cells, the 5'-flanking region showed promoter activity, and the minimal promoter was demonstrated to be located within the -179 to +62 region. Bioinformatics analyses suggested that the minimal promoter contained several canonical Sp1 binding sites. Accumulating evidence shows that $\mathrm{Sp} 1$ is critical for the transcriptional initiation of TATA-less promoters $(27,28)$. Currently, the Sp family consists of nine distinct proteins (Sp1-9) (29), among which Sp1, Sp2, Sp3 and Sp4 comprise two major glutamine-rich transactivation domains, which 
A

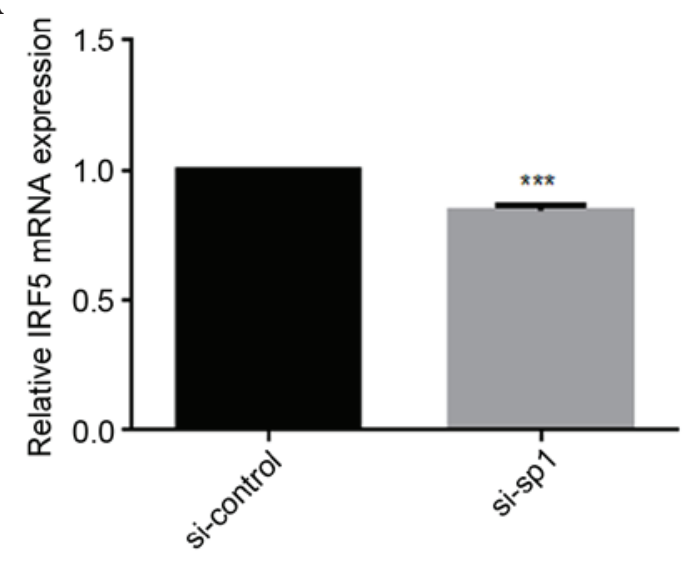

C
B

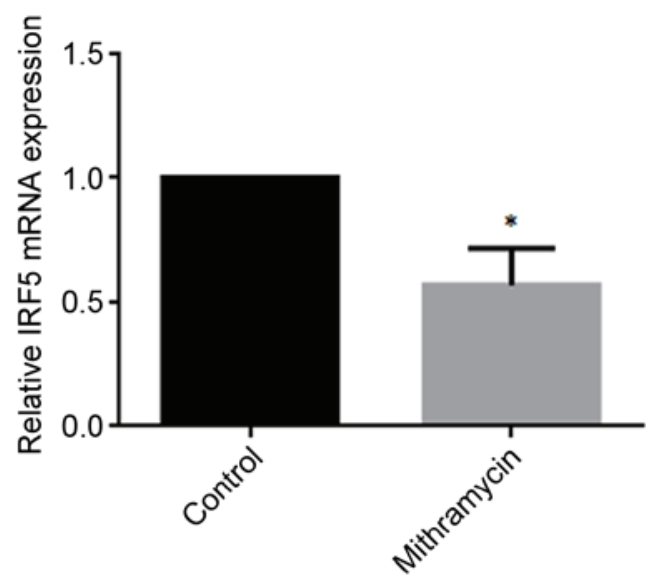

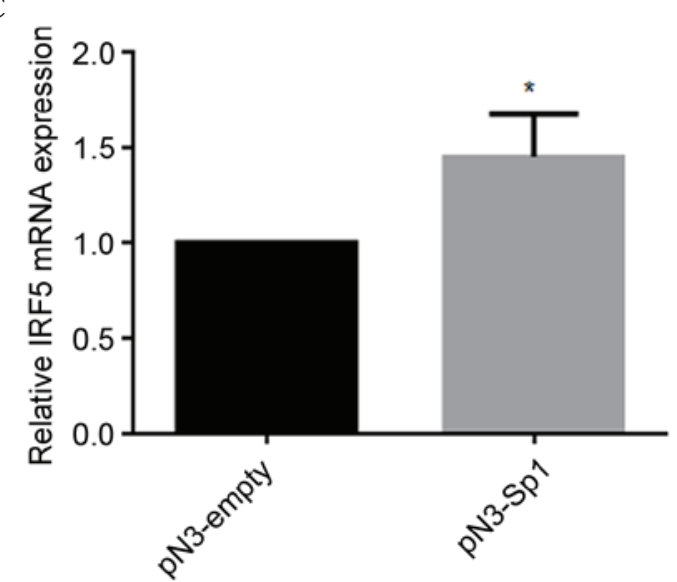

Figure 4. Quantitative polymerase chain reaction analysis of mRNA levels of IRF5 in HEK293 cells. (A) Quantification of mRNA levels of IRF5 follwing siRNA-mediated knockdown of Sp1 ( $\left.{ }^{* * *} \mathrm{P}<0.001\right)$. (B) Quantification of mRNA levels of IRF5 following treatment with mithramycin ("P<0.05). (C) Quantification of mRNA levels of IRF5 following overexpression of pN3-Sp1 ( $\mathrm{P}<0.05)$. All experiments were performed independently in triplicate. IRF5, interferon regulatory factor 5; Sp1, specificity protein 1; siRNA, small interfering RNA.

are essential for transcriptional activation (28). They bind to GC/GT boxes via three $\mathrm{C} 2 \mathrm{H} 2$-type zinc fingers to regulate the expression of housekeeping, tissue-specific and viral genes and are involved in several important aspects of cellular function (30). In the present study, three Spl binding sites were identified within the minimal promoter of IRF5. Of note, all the putative $\mathrm{Spl}$ sites were structurally identical to the consensus binding site (G/T)GGGCGG(G/A)(G/A) $(\mathrm{C} / \mathrm{T})(31)$, although $\mathrm{Sp}-\mathrm{C}$ was located in the reverse strand.

Mutational analyses of the putative $\mathrm{Sp} 1$ sites revealed that individual mutations of the Sp1-A, Sp1-B or Sp1-C sites reduced the activity of the promoter, and the most marked reduction was found in combinatorial mutation of the Sp1-A, Sp1-B and Sp1-C sites. The EMSA and ChIP experiments demonstrated that $\mathrm{Sp} 1$ was able to bind to the promoter of IRF5 in vitro and vivo, respectively. In addition, the overexpression of $\mathrm{Sp} 1$ increased promoter activity and the endogenous mRNA level of IRF5. The present study also identified that knockdown of Sp1 using siRNA technology resulted in a significant decrease in the transcriptional activity of the IRF5 promoter and the mRNA level of IRF5. The present study also showed that treatment of the Hela and HEK 293 cells with mithramycin led to a reduction in the promoter activity and mRNA expression of IRF5. Taken together, these results indicated that Sp1 was essential in the basal transcriptional regulation of IRF5.

Sp1 and Sp3 have the same binding sites, however, whether Sp3 regulates the expression of IRF5 remains to be elucidated. Post-translational modifications of Sp1 and interactions with other transcription factors can also affect transcriptional activity, therefore, the further investigation of the underlying regulatory mechanism is required.

In conclusion, the present study found that the primary activating regulatory region of human IRF5 was located in its minimal promoter region between nucleotides -179 and +62 . In addition, it was shown that $\mathrm{Sp} 1$ was able to bind to the multiple sites in IRF5 promoter region, and was involved in the transcriptional regulation of IRF5 at the basal level. These results may provide novel insight into the molecular mechanisms underlying the regulation of IRF5.

\section{Acknowledgements}

The authors would like to thank Dr G. Suske for the Sp1 expression plasmids. This study was supported by the National Natural Science Foundation of China (grant nos. 81170661 to G.P.Z., 81300023 to R.J. and 81302531 to Hua-Guo Xu), the Natural Science Foundation of Jiangsu Province of China 
(grant nos. BK20131018 to H.G.X. and BK20131020 to R.J.), the Specialized Research Fund for the Doctoral Program of Higher Education (grant no. 20113234110010 to G.P.Z.), and the Project Funded by the Priority Academic Program Development of Jiangsu Higher Education Institutions.

\section{References}

1. Savitsky D, Tamura T, Yanai H and Taniguchi T: Regulation of immunity and oncogenesis by the IRF transcription factor family. Cancer Immunol Immunother 59: 489-510, 2010.

2. Takaoka A, Yanai H, Kondo S, Duncan G, Negishi H, Mizutani T, Kano S, Honda K, Ohba Y, Mak TW and Taniguchi T: Integral role of IRF-5 in the gene induction programme activated by Toll-like receptors. Nature 434: 243-249, 2005.

3. Yanai H, Chen HM, Inuzuka T, Kondo S, Mak TW, Takaoka A, Honda $\mathrm{K}$ and Taniguchi T: Role of IFN regulatory factor 5 transcription factor in antiviral immunity and tumor suppression. Proc Natl Acad Sci USA 104: 3402-3407, 2007.

4. Krausgruber T, Saliba D, Ryzhakov G, Lanfrancotti A, Blazek K and Udalova IA: IRF5 is required for late-phase TNF secretion by human dendritic cells. Blood 115: 4421-4430, 2010.

5. Barnes BJ, Kellum MJ, Field AE and Pitha PM: Multiple regulatory domains of IRF-5 control activation, cellular localization, and induction of chemokines that mediate recruitment of $\mathrm{T}$ lymphocytes. Mol Cell Biol 22: 5721-5740, 2002.

6. Feng D, Stone RC, Eloranta ML, Sangster-Guity N, Nordmark G, Sigurdsson S, Wang C, Alm G, Syvänen AC, Rönnblom L and Barnes BJ: Genetic variants and disease-associated factors contribute to enhanced interferon regulatory factor 5 expression in blood cells of patients with systemic lupus erythematosus. Arthritis Rheum 62: 562-573, 2010.

7. Kim K, Cho SK, Han TU, Kim JH, Kang SJ, Kang C and Bae SC: A redundant epistatic interaction between IRF5 and STAT4 of the type I interferon pathway in susceptibility to lupus and rheumatoid arthritis. Lupus 22: 1336-1340, 2013.

8. Lessard CJ, Li H, Adrianto I, Ice JA, Rasmussen A, Grundahl KM, Kelly JA, Dozmorov MG, Miceli-Richard C, Bowman S, et al: Variants at multiple loci implicated in both innate and adaptive immune responses are associated with Sjögren's syndrome. Nat Genet 45: 1284-1292, 2013.

9. Stone RC, Feng D, Deng J, Singh S, Yang L, Fitzgerald-Bocarsly P, Eloranta ML, Rönnblom L and Barnes BJ: Interferon regulatory factor 5 activation in monocytes of systemic lupus erythematosus patients is triggered by circulating autoantigens independent of type I interferons. Arthritis Rheum 64: 788-798, 2012.

10. Barnes BJ, Kellum MJ, Pinder KE, Frisancho JA and Pitha PM: Interferon regulatory factor 5, a novel mediator of cell cycle arrest and cell death. Cancer Res 63: 6424-6431, 2003.

11. Bi X, Feng D, Korczeniewska J, Alper N, Hu G and Barnes BJ: Deletion of Irf5 protects hematopoietic stem cells from DNA damage-induced apoptosis and suppresses $\gamma$-irradiation-induced thymic lymphomagenesis. Oncogene 33: 3288-3297, 2014.

12. $\mathrm{Hu} \mathrm{G}$ and Barnes BJ: IRF-5 is a mediator of the death receptor-induced apoptotic signaling pathway. J Biol Chem 284: 2767-2777, 2009

13. Massimino M, Consoli ML, Mesuraca M, Stagno F, Tirrò E, Stella S, Pennisi MS, Romano C, Buffa P, Bond HM, et al: IRF5 is a target of BCR-ABL kinase activity and reduces CML cell proliferation. Carcinogenesis 35: 1132-1143, 2014.
14. Yamashita M, Toyota M, Suzuki H, Nojima M, Yamamoto E, Kamimae S, Watanabe Y, Kai M, Akashi H, Maruyama R, et al: DNA methylation of interferon regulatory factors in gastric cancer and noncancerous gastric mucosae. Cancer Sci 101: 1708-1716, 2010.

15. Bi X, Hameed M, Mirani N, Pimenta EM, Anari J and Barnes BJ: Loss of interferon regulatory factor 5 (IRF5) expression in human ductal carcinoma correlates with disease stage and contributes to metastasis. Breast Cancer Res 13: R111, 2011.

16. Massimino M, Vigneri P, Fallica M, Fidilio A, Aloisi A, Frasca F and Manzella L: IRF5 promotes the proliferation of human thyroid cancer cells. Mol Cancer 11: 21, 2012.

17. Kreher S, Bouhlel MA, Cauchy P, Lamprecht B, Li S, Grau M, Hummel F, Köchert K, Anagnostopoulos I, Jöhrens K, et al: Mapping of transcription factor motifs in active chromatin identifies IRF5 as key regulator in classical Hodgkin lymphoma. Proc Natl Acad Sci USA 111: E4513-E4522, 2014.

18. Mancl ME, Hu G, Sangster-Guity N, Olshalsky SL, Hoops K, Fitzgerald-Bocarsly P, Pitha PM, Pinder K and Barnes BJ: Two discrete promoters regulate the alternatively spliced human interferon regulatory factor-5 isoforms. Multiple isoforms with distinct cell type-specific expression, localization, regulation, and function. J Biol Chem 280: 21078-21090, 2005.

19. Graham RR, Kozyrev SV, Baechler EC, Reddy MV, Plenge RM, Bauer JW, Ortmann WA, Koeuth T, González Escribano MF; Argentine and Spanish Collaborative Groups, et al: A common haplotype of interferon regulatory factor 5 (IRF5) regulates splicing and expression and is associated with increased risk of systemic lupus erythematosus. Nat Genet 38: 550-555, 2006.

20. Sigurdsson S, Göring HH, Kristjansdottir G, Milani L, Nordmark G, Sandling JK, Eloranta ML, Feng D, Sangster-Guity N, Gunnarsson I, et al: Comprehensive evaluation of the genetic variants of interferon regulatory factor 5 (IRF5) reveals a novel 5 bp length polymorphism as strong risk factor for systemic lupus erythematosus. Hum Mol Genet 17: 872-881, 2008.

21. Briggs MR, Kadonaga JT, Bell SP and Tjian R: Purification and biochemical characterization of the promoter-specific transcription factor, Sp1. Science 234: 47-52, 1986

22. Livak KJ and Schmittgen TD: Analysis of relative gene expression data using real-time quantitative PCR and the 2(-Delta Delta C(T)) method. Methods 25: 402-408, 2001.

23. Seznec J, Silkenstedt B and Naumann U: Therapeutic effects of the Sp1 inhibitor mithramycin A in glioblastoma. J Neurooncol 101: 365-377, 2011.

24. Xu HG, Jin R, Ren W, Zou L, Wang Y and Zhou GP: Transcription factors Sp1 and Sp3 regulate basal transcription of the human IRF-3 gene. Biochimie 94: 1390-1397, 2012.

25. Ren W, Zhu LH, Xu HG, Jin R and Zhou GP: Characterization of a spliced variant of human IRF-3 promoter and its regulation by the transcription factor Sp1. Mol Biol Rep 39: 6987-6993, 2012.

26. Clark DN, Read RD, Mayhew V, Petersen SC, Argueta LB, Stutz LA, Till RE, Bergsten SM, Robinson BS, BaumannDG, et al: Four promoters of IRF5 respond distinctly to stimuli and are affected by autoimmune-risk polymorphisms. Front Immunol 4: 360, 2013.

27. Kadonaga JT, Carner KR, Masiarz FR and Tjian R: Isolation of cDNA encoding transcription factor $\mathrm{Spl}$ and functional analysis of the DNA binding domain. Cell 51: 1079-1090, 1987.

28. Suske G: The Sp-family of transcription factors. Gene 238: 291-300, 1999.

29. Suske G, Bruford E and Philipsen S: Mammalian SP/KLF transcription factors: Bring in the family. Genomics 85: 551-556, 2005.

30. Philipsen S and Suske G: A tale of three fingers: The family of mammalian Sp/XKLF transcription factors. Nucleic Acids Res 27: 2991-3000, 1999.

31. Tan NY and Khachigian LM: Sp1 phosphorylation and its regulation of gene transcription. Mol Cell Biol 29: 2483-2488, 2009. 